\title{
Core magnetic islands and plasma confinement in the H-1NF heliac
}

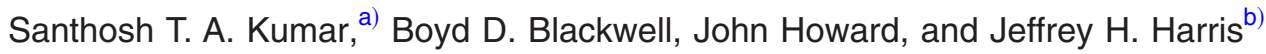 \\ Plasma Research Laboratory, Research School of Physical Sciences and Engineering, \\ The Australian National University, Canberra, ACT 0200, Australia
}

(Received 15 March 2010; accepted 13 July 2010; published online 13 August 2010)

\begin{abstract}
Plasma confinement in the vicinity of vacuum magnetic islands near the magnetic axis in the $\mathrm{H}-1 \mathrm{NF}$ heliac [S. M. Hamberger et al., Fusion Technol. 17, 123 (1990)] has been experimentally studied in a low temperature argon plasma. Experimental results indicate that, under favorable conditions, these low order $(m=2)$ islands near the core of the plasma serve as "pockets" of higher electron density. This results in significant profile modifications including enhancement of the core radial electric field to a large positive value, possibly through an electron-root ambipolar condition. The characteristics of islands are found to be dependent on the plasma collisionality and island width. (C) 2010 American Institute of Physics. [doi:10.1063/1.3474946]
\end{abstract}

\section{INTRODUCTION}

Rational magnetic flux surfaces in toroidal plasma confinement devices can break the magnetic field lines and reconnect them in the form of magnetic islands. Formation of these magnetic islands can have a serious impact on the plasma confinement properties of the device, depending on both plasma and island parameters. Islands generally degrade plasma confinement by mixing up different regions of plasma. However, there is experimental evidence that islands can improve the confinement under favorable conditions. For example, the role of edge islands in the formation of edge thermal transport barriers and transition to improved confinement regime (H-mode) in the Large Helical Device ${ }^{1}$ (LHD) has been experimentally confirmed. ${ }^{2}$ Rational surfaces and magnetic islands near the core of the plasma are thought to be responsible for the generation of, and lowering the threshold conditions for generating, the "core electron-root confinement" (CERC) in helical devices.,

Islands in plasma configurations have been inferred from the "flattening" of the radial profiles of the plasma temperature or density (for example, see Refs. 5 and 6). In LHD, it has been observed that there is a minimum size of the island, below which profile flattening does not occur. ${ }^{7}$ This was interpreted in terms of the balance between cross-field and parallel transport. Flattening of the radial profile may be attributed to the poor confinement inside the island or to the poor resolution of diagnostics. However, in a separate experiment on LHD, it has been observed that the heat transport inside an island is much reduced, ${ }^{8}$ which indicates that the profile flattening does not necessarily imply deterioration of the confinement within the island. Further, a local density peak inside magnetic islands has been observed in some tokamak discharges. ${ }^{9,10}$ The parametric conditions required for observing any of these island signatures remain unclear.

A simple hypothesis is in terms of the relation between the island width, gyroradius of the particles, collision scale

\footnotetext{
${ }^{a)}$ Present address: Department of Physics, University of Wisconsin-Madison, USA. Electronic mail: stkumar@wisc.edu.

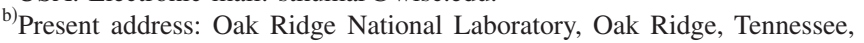
USA.
}

lengths, and the "half connection length" (the toroidal distance from one end of the island to the other) of the island. The $\nabla P=0$ condition on a flux surface, where $P$ is the plasma pressure, in principle requires the parameters to be the same on both sides of an island, if collisionality conditions are satisfied: If the collision mean free path is much less than the half connection length of the island, the probability of observing same plasma conditions on both sides of the island is less. Also, if the gyroradius of particles is more than the half width of the island $(\delta / 2)$, particles do not "feel" the island: the kinetic effects may "smooth out" any profile modification that would occur otherwise. A more complete picture should include drifts and trapped particle effects.

This paper reports some experimental results on plasma confinement in the vicinity of the core vacuum magnetic islands in the H-1NF. Islands are observed to behave as improved confinement regions with a density peak near the island O-point, under favorable conditions. (Our claim of improved confinement inside the island is based on only the local density gradients. A proper quantification of confinement improvement, in terms of the particle or thermal diffusivities, is beyond the scope of this paper.) Possible mechanisms are discussed, including the electron-root ambipolar conditions which have previously been observed only in very high temperature plasmas.

\section{EXPERIMENTAL SETUP}

Experiments were conducted on the H-1NF heliac, which is a medium sized helical axis stellarator ${ }^{11}$ with major radius of $\sim 1 \mathrm{~m}$ and average plasma minor radius of $\sim 0.2 \mathrm{~m}$. The coil system of $\mathrm{H}-1 \mathrm{NF}$ allows the configuration to be varied across a broad range, $0.6<t<2.0$, where $t=n / m$ is the rotational transform and $n$ and $m$ are the toroidal and poloidal winding numbers, respectively, by changing coil current ratios. This allows systematic control of the presence and position of the major rational surfaces and islands in the main confinement volume. ${ }^{12}$ Vacuum mapping of flux surfaces and islands has been carried out recently using electron beam wire tomography ${ }^{13}$ and an accurate computer model for H-1NF magnetic geometry has been developed. ${ }^{14}$ 

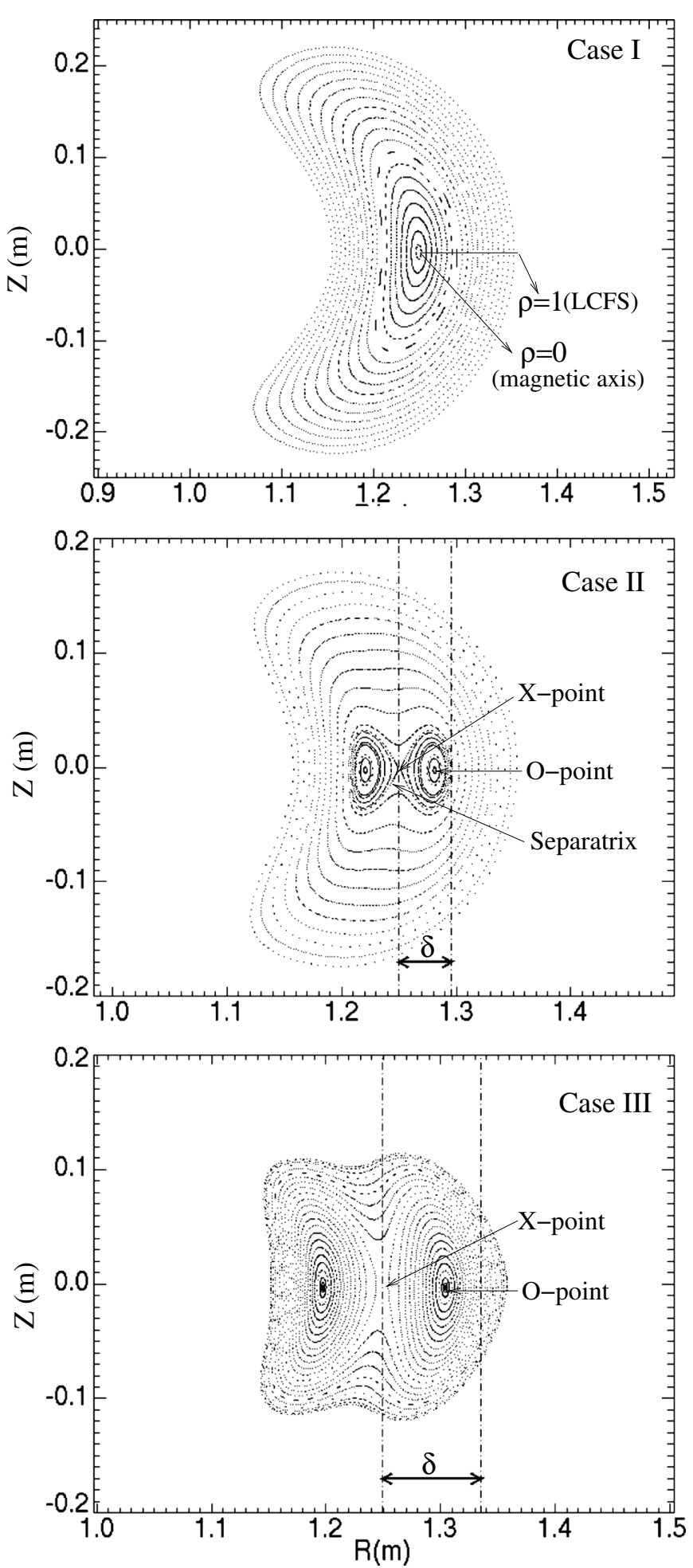

FIG. 1. Computed flux surfaces of the three configurations used for study. $\delta$ is the width of the vacuum magnetic island. The islands (Case II and III) are toroidally connected.

Low order $m=2(t=3 / 2)$ islands inherent to the helical configuration of the H-1NF provide an excellent opportunity for island studies. These islands are relatively easily observed in vacuum configurations and the size of the island and their position in minor radius can be varied in a controlled fashion. Three magnetic configurations (shown in Fig. 1) have mainly been used for experiments reported in this paper.
- Case I: ( $t \sim 1.35-1.38)$ This configuration has well-nested (unperturbed) magnetic flux surfaces as shown in Fig. 1. Profiles obtained from this configuration are used for comparison with that of the island configurations.

- Case II: $(t \sim 1.44-1.50)$ This configuration exhibits vacuum magnetic islands with width $\delta \sim 0.4 \rho(\sim 40 \mathrm{~mm})$, where $\rho=\mathrm{r} / \mathrm{a}$, the radial distance from the magnetic axis normalized to the radial location of the last closed flux surface $(\mathrm{r}=\mathrm{a})$ as shown in Fig. 1.

- Case III: For this configuration, the vacuum islands $(t=3 / 2)$ cover most of the plasma region with width $\delta \sim 0.8 \rho$, results in a (toroidally connected) "doubletlike" configuration.

Experiments have been conducted in a low magnetic field ( $\sim 0.1 \mathrm{~T}$ at the axis) argon plasma, produced by radio frequency (rf) helicon waves of power of $\sim 60 \mathrm{~kW}$ for a discharge duration of 40-60 ms. Typical chamber fill pressure for the discharges is $3.0 \times 10^{-5}$ Torr, although this is varied where noted. A radially movable Langmuir probe (tip length of $\sim 1.4 \mathrm{~mm}$ and diameter of $\sim 0.5 \mathrm{~mm}$ ) has been used to obtain local plasma parameters. The probe position has been calibrated using an electron beam in vacuum. A more accurate determination of the magnetic axis is obtained later on from the symmetry point of the density profile of a configuration without islands. The low electron temperature $(\sim 10 \mathrm{eV})$ of these low magnetic field argon plasmas allows the use of Langmuir probes. Argon plasmas in H-1NF are highly reproducible and less affected by the insertion of probes. ${ }^{15}$ The swept probe is biased with a sine wave of frequency of $30 \mathrm{kHz}$ and bias voltage up to $\pm 150 \mathrm{~V}$. Plasma parameters $\left(n_{e}, T_{e}, \phi_{f}\right)$ are thus temporally $(\Delta t \sim 16 \mu \mathrm{s})$ and spatially $(\Delta r=5 \mathrm{~mm})$ resolved. Plasma potential $\left(\phi_{p}\right)$ is calculated from the floating potential $\left(\phi_{f}\right)$ using the relation $\phi_{p} \sim \phi_{f}+4.7 T_{e} .{ }^{16}$ Electric field is calculated $\left(E_{r}=-\nabla \phi_{p}\right)$ from the fitted curve of $\phi_{p}$. Radial profiles are averaged over two sets of measurements and the error bars in density and plasma potential are not bigger than the size of the symbols shown in the figures. A reference Langmuir probe has been positioned near the edge of the plasma (last closed flux surface) to monitor any perturbation in the plasma parameters due to the insertion of the probe.

A coherent imaging camera ${ }^{17}$ has been used to obtain the spectral light intensity from plasma radiation. The spectral light intensity (I) of the plasma radiation from singly charged ion has a simple functional dependency on the plasma density $\left(n_{e}\right)$ and the electron temperature $\left(T_{e}\right),{ }^{18}$

$$
I \propto n_{e}^{2} \xi\left(T_{e}\right),
$$

where $\xi$ is the distribution averaged rate coefficient for collisional excitation from the ground state. Evidently, the electron density has a strong effect on the spectral light intensity. Assuming that the temperature does not vary much during a discharge (for example, see Fig. 4), the spectral intensity can give a direct indication of the plasma density. It has to be noted that spectroscopic measurements are line of sight averaged, unlike the Langmuir probe measurements which are localized. The camera was used to estimate the bulk rotation velocity from Doppler shift of the Ar II line at 


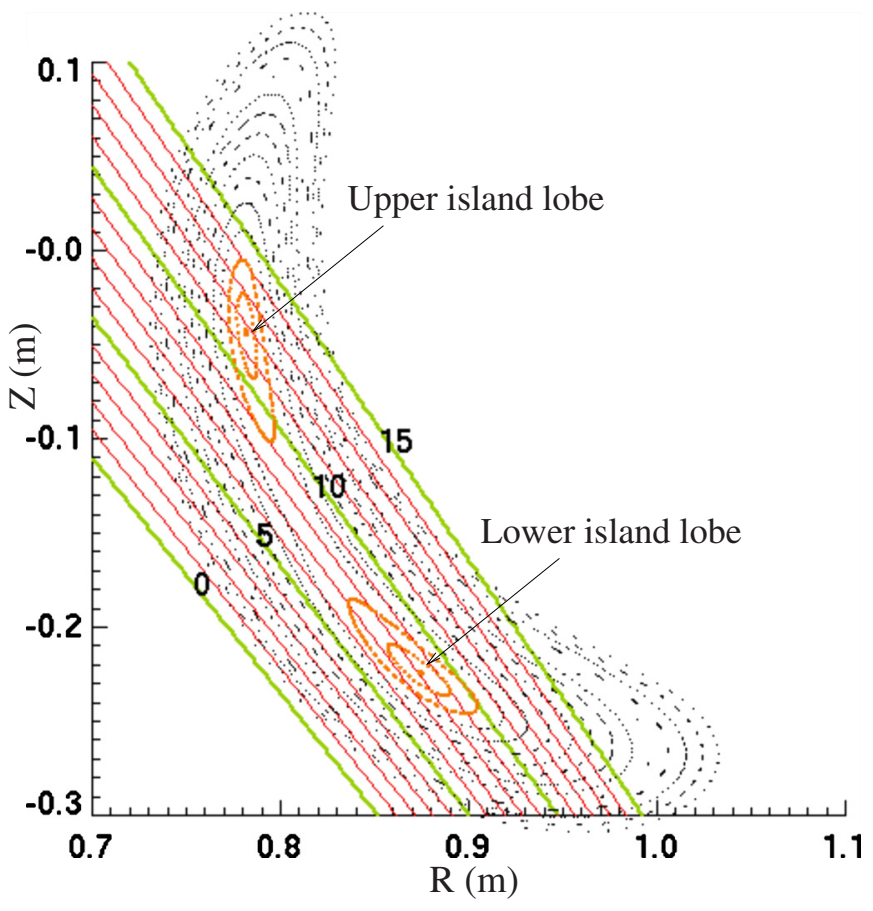

FIG. 2. (Color online) Viewing chord positions for the 16 channel imaging camera, overlayed on the flux surfaces from the configuration exhibiting $m=2$ vacuum magnetic islands (Case II). Channel numbers (0-15) are shown.

$\lambda=488 \mathrm{~nm},{ }^{17-19}$ and with the coherence feature disabled, to measure the light intensity. The lines of sight (viewing chords) for this 16 channel camera, calibrated using an electron beam, are illustrated in Fig. 2.

\section{EXPERIMENTAL RESULTS}

Radial profiles of plasma parameters for the three configurations are shown in Figs. 3-5, respectively. Major experimental observations can be summarized as follows.

- Case I: The electron temperature has a "flat" profile $\left(T_{e}\right.$ $\sim 8 \mathrm{eV}$ ) for most of the plasma minor radius with an increase at the edge. This is the typical behavior of $T_{e}$ for the rf argon discharges of $\mathrm{H}-1 \mathrm{NF}^{20}$ A plasma density of $\sim 1.5 \times 10^{18} / \mathrm{m}^{3}$ has been obtained near the core with a centrally flat profile that decreases monotonically toward the edge. The plasma potential is almost flat near the core and increases toward the edge. The radial electric field calculated from the plasma potential is in the range from 0 to $-4 \mathrm{kV} / \mathrm{m}$.

- Case II: Magnetic islands manifest in the form of density peaking close to the island O-point (Fig. 4), throughout the discharge, even though there is a decrease in density globally (which is somewhat expected, as the plasma confinement in $\mathrm{H}-1 \mathrm{NF}$ is highly sensitive to the presence of rational surfaces/islands ${ }^{21}$ ). The electron temperature is flat $\left(T_{e} \sim 10 \mathrm{eV}\right)$ at the core and increases toward the edge. The plasma potential has a dip near the island O-point. The resultant electric field becomes positive $\left(E_{r} \sim 2-4 \mathrm{kV} / \mathrm{m}\right)$ near the core with a large electric field shear $\left(\sim 300 \mathrm{kV} / \mathrm{m}^{2}\right)$ inside the island with a shear layer thickness of $\sim \delta / 2$, where $\delta$ is the full width of the island. The
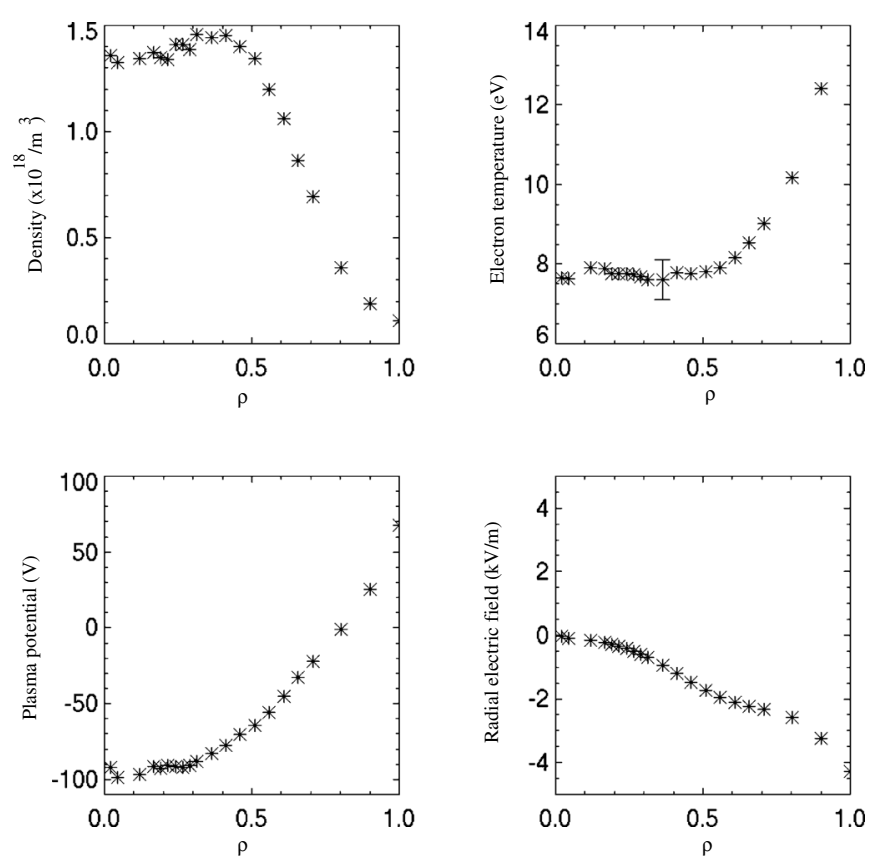

FIG. 3. Radial profiles of plasma density $\left(n_{e}\right)$, electron temperature $\left(T_{e}\right)$, plasma potential $\left(\phi_{p}\right)$, and radial electric field $\left(E_{r}\right)$ for the no-island configuration (Case I) at $\sim 20 \mathrm{~ms}$ into the discharge.

$E_{r}$ has a minimum point inside the island which coincides with the island O-point. The dip in the $E_{r}$ at $\rho \sim 0.8-0.9$ corresponds to the density gradient. A similar behavior is observed when the neutral density (chamber fill pressure)
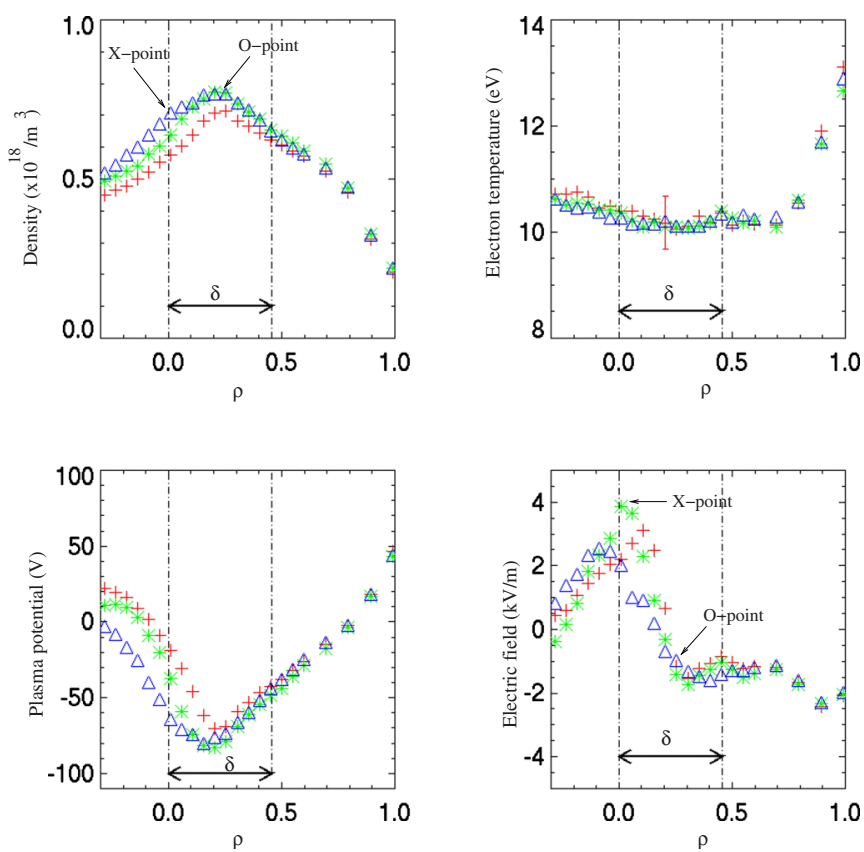

FIG. 4. (Color online) Radial profiles of plasma density $\left(n_{e}\right)$, electron temperature $\left(T_{e}\right)$, plasma potential $\left(\phi_{p}\right)$, and electric field $\left(-\nabla \phi_{p}\right)$ for the island configuration (Case II) at $\sim 10 \mathrm{~ms}(+), \sim 20 \mathrm{~ms}\left({ }^{*}\right)$, and $\sim 30 \mathrm{~ms}(\triangle)$ into the discharge. $\delta$ is the width of the vacuum magnetic island. (Note that the radial coordinate axis is extended to negative values to explore symmetries. The electric field is plotted as if the coordinates were Cartesian, and therefore would be expected to have odd symmetry about $\rho=0$. The asymmetry about $\rho=0$ is discussed in Sec. IV.) 

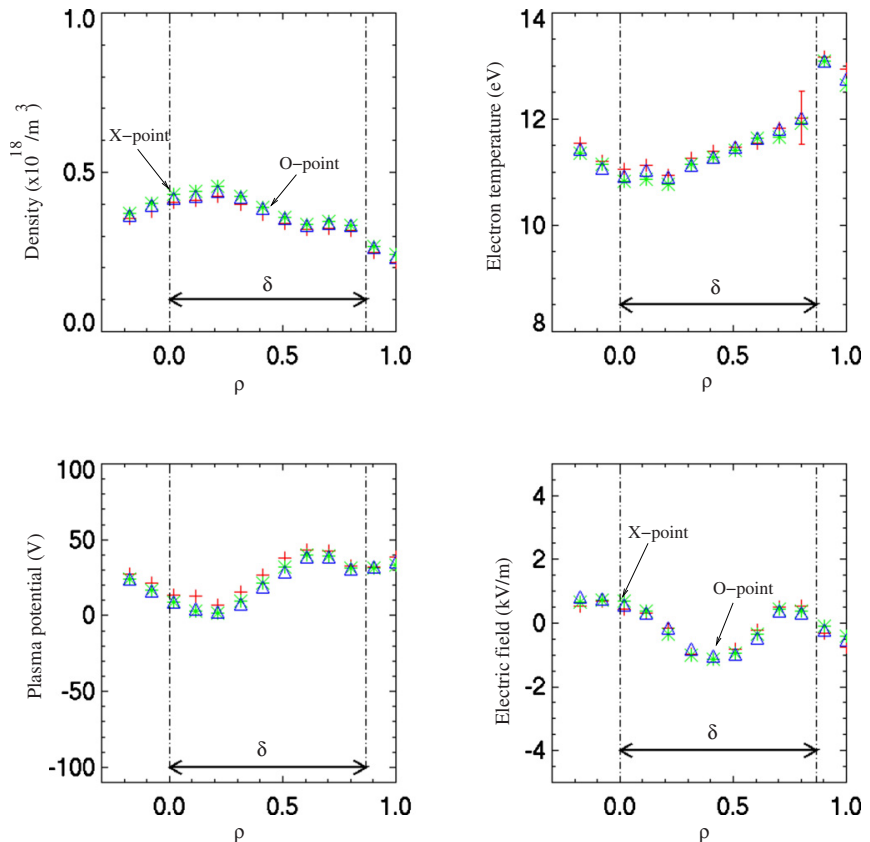

FIG. 5. (Color online) Radial profiles of plasma density $\left(n_{e}\right)$, electron temperature $\left(T_{e}\right)$, plasma potential $\left(\phi_{p}\right)$, and electric field $\left(-\nabla \phi_{p}\right)$ for the doublet configuration (Case III) at $\sim 10 \mathrm{~ms}(+), \sim 20 \mathrm{~ms}(*)$, and $\sim 30 \mathrm{~ms}(\triangle)$ into the discharge. $\delta$ is the width of the vacuum magnetic island.

is halved. However, when neutral pressure is doubled, these potential, density, and electric field structures disappear as shown in Fig. 6.

- Case III: For this doublet configuration, the plasma confinement is poor, as indicated by the low plasma density (Fig. 5). Nearly 50\% decrease in the plasma density has been observed. The density profile in this case does not peak at the island O-point, as observed in Case II, but exhibits two inflections $(\rho=0.4,0.85)$ with a peak near the magnetic axis. The $T_{e}$ increases toward the edge with a core value of $T_{e} \sim 11 \mathrm{eV}$ and edge $T_{e} \sim 13 \mathrm{eV}$. The
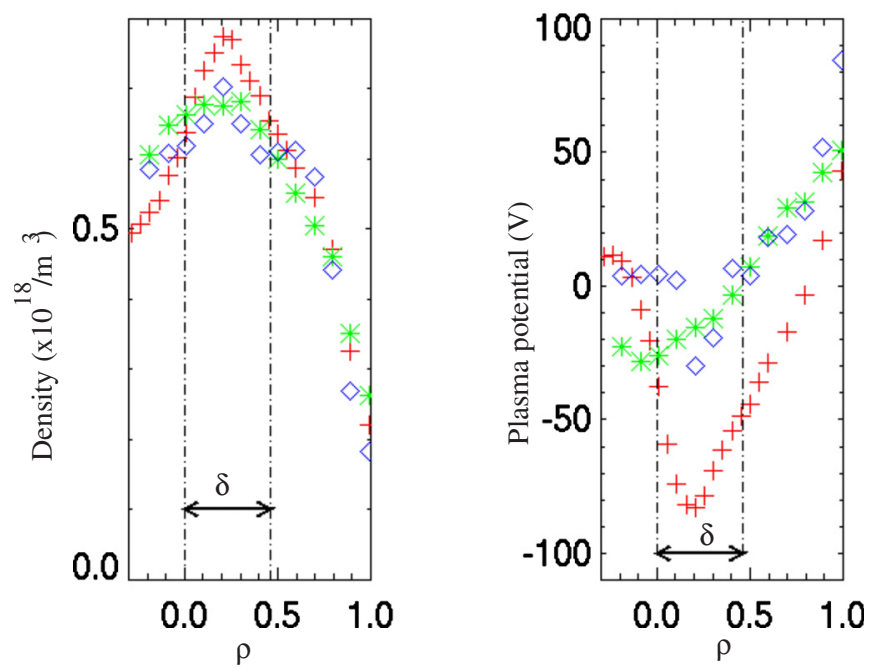

FIG. 6. (Color online) Radial profiles of plasma density and plasma potential for the island configuration (Case II) at three different neutral pressures. $(+$ : Normal neutral pressure; *: double neutral pressure; $\diamond$ : half neutral pressure.)

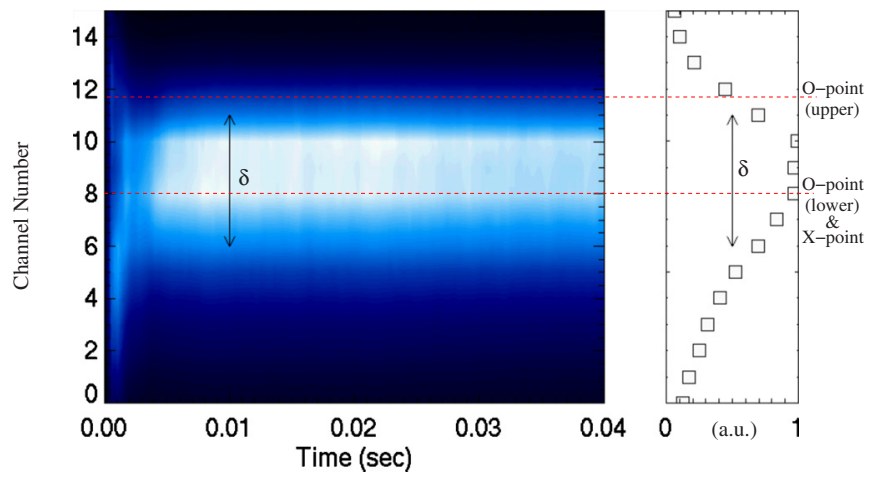

FIG. 7. (Color online) The spectral light intensity (normalized to the maximum brightness) for a configuration exhibiting vacuum islands (Case II), showing the lower island lobe is brighter throughout the discharge. $\delta$ is the approximate width of one of the vacuum island lobes (the lower island lobe in Fig. 2). Plotted on the right side is the normalized brightness (arbitrary units) at $\sim 20 \mathrm{~ms}$ into the discharge, and the O-points are shown. (See Fig. 2 for viewing chords.)

plasma potential is $\sim 10 \mathrm{~V}$ near the core, and increases toward the edge of the plasma to just past the island O-point, and remains rather flat after that. The resultant $E_{r}$ has a minimum point $(\sim-1.5 \mathrm{kV} / \mathrm{m})$ which coincides with the island O-point and has a symmetry around the O-point (Fig. 5).

The spectral light intensity contours obtained by the coherent imaging camera, for the configuration exhibiting vacuum islands (Case II), are shown in Fig. 7. The density peak inside the island, throughout the discharge, is apparent from the Fig. 7. It can also be noted that one of the two $m$ $=2$ island lobes is much brighter than the other, which will be discussed in Sec. IV.

\section{DISCUSSION}

The typical plasma parameters for the discharges mentioned above (except the high neutral pressure discharges) are listed in Table I. It can be seen that, for the experimental conditions, $\omega_{c i} \lesssim \nu_{e i} \ll \omega_{c e}$, where $\omega_{c i}, \nu_{e i}$, and $\omega_{c e}$ are the ion gyrofrequency, electron-ion collision frequency, and electron gyrofrequency, respectively. Electrons are therefore highly magnetized and ions are nearly magnetized. Also, for Case II, $\rho_{e} \ll \delta / 2<\rho_{i}$, where $\rho_{e}, \rho_{i}$, and $\delta$ are the electron and ion gyroradii and the island width, respectively. Therefore, ion dynamics is not much affected by the presence of island (as $\rho_{i}>\delta / 2$, ions do not feel the presence of these islands).

The radial electric field can be calculated from the radial force balance equation, ${ }^{25}$

$$
E_{r}=\frac{\nabla P_{i}}{n_{i} Z_{i} e}-\left(v_{\theta} B_{\phi}-v_{\phi} B_{\theta}\right),
$$

where $B_{\phi}$ and $B_{\theta}$ are the toroidal and poloidal magnetic fields, $v_{\phi}, v_{\theta}$ are toroidal and poloidal plasma rotation velocities, and $Z_{i} e, n_{i}$, and $P_{i}$ are ion charge, density, and ion pressure, respectively. In the absence of plasma rotation, the radial electric field can be approximated to the ion pressure gradient term, $\nabla P_{i} / n_{i} Z_{i} e$. Figure 8 compares the $E_{r}$ profile calculated from the pressure gradient $\left(\nabla P_{i} / n_{i} Z_{i} e\right)$ to the $E_{r}$ 
TABLE I. Typical plasma parameters for the usual neutral pressure discharge.

\begin{tabular}{lc}
\hline Electron temperature & $10 \mathrm{eV}$ \\
Ion temperature $^{\mathrm{a}}$ & $\sim 30-80 \mathrm{eV}$ \\
Neutral (fill) density $^{\mathrm{b}}\left(n_{n}\right)$ & $\sim 0.81 \times 10^{18} / \mathrm{m}^{3}$ \\
Plasma density & $\sim 1 \times 10^{18} / \mathrm{m}^{3}$ \\
Electron thermal velocity $\left(V_{\text {the }}\right)$ & $1.3 \times 10^{6} \mathrm{~m} / \mathrm{s}$ \\
Ion acoustic velocity $\left(C_{s}\right)$ & $1 \times 10^{4} \mathrm{~m} / \mathrm{s}$ \\
Electron gyro radius $\left(\rho_{e}\right)$ & $0.075 \mathrm{~mm}$ \\
Ion gyro radius $\left(\rho_{i}\right)$ & $\sim 35-55 \mathrm{~mm}$ \\
Electron gyro frequency $\left(\omega_{c e}\right)$ & $1.7 \times 10^{10} \mathrm{rad} / \mathrm{s}$ \\
Ion gyro frequency $\left(\omega_{c i}\right)$ & $2.5 \times 10^{5} \mathrm{rad} / \mathrm{s}$ \\
Electron-ion collision frequency $\left(\nu_{e i}\right)$ & $9.2 \times 10^{5} / \mathrm{s}$ \\
Electron-neutral collision frequency $\left(\nu_{e n}\right)$ & $1.6 \times 10^{5} / \mathrm{s}$ \\
Collision mean free path $\left(\lambda_{e i}\right)$ & $\sim 2.5 \mathrm{~m}$ \\
Collision mean free path $\left(\lambda_{e n}\right)$ & $\sim 8.2 \mathrm{~m}$ \\
\hline
\end{tabular}

${ }^{\mathrm{a}}$ From previous measurements on similar discharges (Ref. 20). $T_{i}=30 \mathrm{eV}$ is taken for most of the calculations, unless otherwise mentioned.

${ }^{\mathrm{b}}$ The background neutral density is expected to drop by $\sim 1 \%$ by virtue of plasma pumping (Ref. 22). The central neutral density (in plasma) depletes to $\sim 30 \%$ of the background neutral density (Refs. 19 and 23 ).

${ }^{c} \nu_{e n}=n_{n} \sigma V_{\text {the }}$, where $\sigma$ is the momentum transfer collision cross-section. $\sigma=1.5 \times 10^{-15} \mathrm{~cm}^{2}$ for $10 \mathrm{eV}$ electrons (Ref. 24).

obtained from the Langmuir probe, for Case II. It can be shown that the dip in $E_{r}$ observed at $\rho \sim 0.8-0.9$ for Case II can be explained in terms of the pressure gradient, when a radially flat ion temperature of $60 \mathrm{eV}$ is assumed. (We do not have measurements of ion temperature for this experiment, but ion temperature measured previously for similar discharges is $\sim 30-80 \mathrm{eV} .{ }^{20}$ Doppler measurements under similar conditions ${ }^{18,19}$ indicate a two component ion distribution with a smaller cold component $[\sim 20 \%,<10 \mathrm{eV}]$ and a majority ion temperature of $\sim 30 \mathrm{eV}$ near the core and

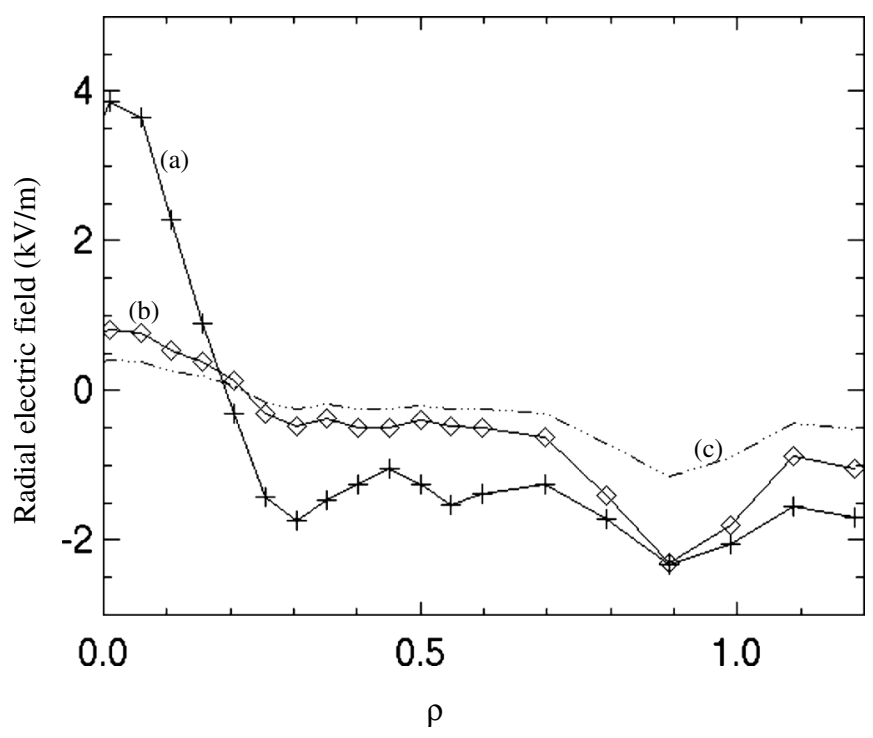

FIG. 8. The radial electric field for the island configuration (Case II), calculated from the pressure gradient using a radially flat ion temperature of 60 $\mathrm{eV}(\mathrm{b})$ and $30 \mathrm{eV}$ (c), over plotted on the measured $E_{r}$ (a). The nonzero $E_{r}$ on axis is due to the asymmetry of the potential profile. In an ideal situation where both island lobes are equally populated, $E_{r}$ would vanish on axis, maintaining positive value between island O-point and magnetic axis. $\sim 60 \mathrm{eV}$ near the edge.) However, the discrepancy between $E_{r}$ calculated as above and measurement and the sharp gradient near the core in Case II suggest that rotation is not negligible, in comparison to $\nabla P$. We posit that an $\mathrm{E} \times \mathrm{B}$ shear flow is set up inside the island, the sign of which reverses (in the coordinate system shown in Fig. 8) across the center of the island as the sign of the electric field reverses. This observation is in line with the previous observation of vortexlike plasma flow inside an island in LHD, ${ }^{26}$ although the plasma parameters are quite different. It is not surprising that the line averaged rotation velocity obtained from the Doppler imaging camera $\left(v_{\theta} \sim 1000-2000 \mathrm{~m} / \mathrm{s}\right)$ is much lower than the expected $\mathrm{E} \times \mathrm{B}$ velocity (in the range of $-2 \times 10^{4}$ to $4 \times 10^{4} \mathrm{~m} / \mathrm{s}$ ). The camera may not resolve the contribution of the island to the line of sight because of the averaging nature of the line integral and some averaging over the viewing volume. The bulk poloidal velocity contribution to $E_{r}$ may explain some of the offset in Fig. 8 away from the axis $(0.4<\rho<0.7)$.

We consider two ways in which the radial electric field can be modified due to the presence of islands. (a) The flows associated with the ambipolar condition $\Gamma_{i}=\Gamma_{e}$, where $\Gamma_{i}$ and $\Gamma_{e}$ are radial fluxes of ions and electrons, respectively, is significantly modified in the vicinity of magnetic islands. As the island radially connects different regions of the plasma, parallel transport in the vicinity of the island (especially near the separatrix) eventually results in radial flux. For the vacuum island configuration (Case II), the island X-point is close to the position of the magnetic axis in the absence of the $3 / 2$ perturbation. As the ion gyroradius $\left(\rho_{i}\right)$ is more than the half width of the island, the ion dynamics (and therefore, $\Gamma_{i}$ ) are likely to be less affected by the presence of island. Therefore, an enhanced preferential flux of electrons is expected from the core of the plasma due to the rapid electron transport along the magnetic field lines near the island boundary, especially from within one $\rho_{e}$ of the island separatrix. Plasma could thus set up a positive radial electric field so that the electron and ion flux are balanced. However, it has to be noted that due to the high collisionality in our experimental conditions and the extremely long halfconnection length near the X-point region $(\sim 10-50$ toroidal transits), electrons from the $\mathrm{X}$-point are very unlikely to reach the other side of the island before making a collision. Even though this effect is expected to play an important role only at lower neutral pressures or long mean-free-path regimes, a preferential loss of electrons from the X-point region is still expected to create a charge separation in our experimental conditions. Our field line trace based on the most realistic model of error fields does not show any significant ergodic region in the vicinity of the separatrix, which under different circumstances might have provided another path for the rapid electron loss from the X-point region. As the X-point is very sensitive to perturbations (drift effects, electric field effects, etc.), there may be some other mechanisms which can take electrons out of this region. (b) The flux surfaces inside the island are still well-nested. Therefore, these islands would behave as regions of good electron confinement leading to the development of a potential well inside the island. This modifies the radial electric field. In our 
experimental conditions, the radial profiles are modified to a large extent by the good electron confinement inside the island and to some extent by the "leaky" X-point. It also should be noted that because the primary diagnostic is ion density (electron density) from a Langmuir probe, our measurements apply directly only to particle confinement.

Positive radial electric field due to the presence of magnetic islands has been observed elsewhere also. For example, in LHD, a nonflat space potential and positive radial electric field are observed when the low order $(\mathrm{n} / \mathrm{m}=1 / 1)$ externally imposed magnetic islands become large enough. ${ }^{26,27}$ Positive radial electric field due to magnetic islands has also been observed in tokamaks. ${ }^{28}$ It has also been theoretically predicted that in the vicinity of a magnetic island in a tokamak, the radial electric field can bifurcate to a large value. ${ }^{29}$

When the neutral density is doubled (from the usual value of $\sim 0.81 \times 10^{18} / \mathrm{m}^{3}$ ) for Case II, the electron neutral collision frequency (momentum transfer) doubles ( $\nu_{\mathrm{en}}$ $\sim 3.2 \times 10^{5} / \mathrm{s}$ ). There are two effects due to this. ${ }^{30}$

(a) The perpendicular collisional (classical) diffusion increases as the diffusion coefficient $D_{\perp} \propto \nu$. This results in degradation of the electron confinement within the island.

(b) The parallel transport of electrons along the magnetic field lines near the separatrix (which is essentially the $\Gamma_{e}$ because of the radial connection of the island) is much reduced due to the increased collisions with neutrals, as the parallel diffusion coefficient $D_{\|} \propto 1 / \nu$.

As a result, the structures in the density, potential, and electric field disappear. This is well supported by our experimental observations (see Fig. 6). When the neutral density is halved, the potential and density structures are still present (although the global values are different), as shown in Fig. 6. This indicates that island signatures depend on collisionality. However, our simple hypothesis in terms of collision scale lengths and the half connection length of the island may not be valid, as explained later in this section.

When the half width of the island becomes much larger than the ion gyroradius as in Case III, $\left(\rho_{e}, \rho_{i} \ll \delta / 2\right)$, there is no preferential diffusion due to the presence of island. The situation is not very different from the no-island case as far as the transport process is concerned. This is in good agreement with the experimental observations of much reduced $E_{r}$ in Case III. A decrease in plasma density by a factor of $\sim 2$ is observed in Case III, even though the plasma volume in this configuration is not different from other cases. This may indicate that the rf ionization process is affected by the presence of islands in vacuum configuration.

As mentioned before, it has been observed, both from the Langmuir probe results and from the spectroscopy, that the radial profiles for the island configurations (Case II) are not symmetric about the magnetic axis (for example, see Fig. 7 where only one lobe of the island is "bright"). Even though the two $m=2$ island lobes are toroidally connected, one of the island lobes is relatively dense. (It can be shown, by tracing the field line, that Figs. 4 and 7 agree as to which island lobe is stronger.) Although not well understood, a similar imbalance was observed in previous experiments in a related magnetic configuration. ${ }^{31,32}$ The asymmetry of $\mathrm{H}-1 \mathrm{NF}$ rf discharges is much discussed in the past. For example, it has been reported that the argon light intensity and density profiles often violate flux symmetry on $\mathrm{H}-1 \mathrm{NF}$ discharges. ${ }^{18}$ This asymmetry was attributed to be driven by the helicon wave heating mechanism which is known to produce asymmetric discharges due to capacitive coupling of the input power. ${ }^{33}$

By tracing the field line back to the rf antenna port, it can be shown that the two island lobes are approximately at equal distance from the rf antenna. Due to subtle effects (for example, at the initial phase of the discharge, the power coupling from the antenna is mainly capacitive, which is known to be asymmetric; a slight misalignment of the antenna may make one of the island lobes closer to the antenna which could exacerbated the issue), an imbalance in the plasma density may be occurring at the initial phase of the discharge. As the flux surfaces inside the magnetic islands are wellnested, plasma confinement inside the island is good. As both the dielectric constant and collision rate increase with electron density, for wave heating, ionization may be more effective in the regions of higher density (at the O-point of the stronger island lobe), reinforcing any imbalance at the initial stages of the discharge and leading to much higher density in one island lobe. As the electron-neutral collision mean-freepath is less than or comparable to one toroidal transit under our experimental conditions, this imbalance is unlikely to be corrected by toroidal connection. Further, if the ionization is more effective at the O-point, the density profile inside the island is expected to be symmetric around the island O-point (see Fig. 4), even though the collisionality conditions for $\nabla P=0$ on a flux surface are not satisfied: both the electronion and electron-neutral collision mean-free-paths $\left(\lambda_{e i}\right.$ and $\left.\lambda_{e n}\right)$ are less than or comparable to one toroidal transit, and therefore, much less than the half connection length of the island (10-12 toroidal transits, which is about 70-90 m). Cross-field diffusion with a source peaked at the O-point determines the density profile inside the island.

A positive radial electric field near the core of the plasma and associated enhancement of plasma confinement in helical devices have recently been classified as CERC. ${ }^{4}$ However, this has been observed only in high temperature electron cyclotron heated $(\mathrm{ECH})$ plasmas which satisfy some threshold conditions (conditions on ECH power per particle). Rational surfaces and magnetic islands near the core of the plasma are thought to be responsible for the generation of, and lowering the threshold conditions for generating, CERC in helical devices. ${ }^{3,4,34}$ Our experimental results may indicate that core magnetic islands could help in achieving the CERC equivalent scenario in low temperature rf plasmas as well.

\section{CONCLUSION}

This paper presents a detailed experimental study of plasma confinement in the vicinity of the core "natural" $m$ $=2$ vacuum magnetic islands in the H-1NF heliac. Our results show that these islands behave as localized regions of higher electron density, under favorable conditions. A model has been put forward to explain the radial profile modifica- 
tion by the island, especially the radial electric field: when the ion gyroradius is larger than or comparable to the half width of the island, the ion dynamics are less affected by the presence of island, but the electron dynamics are significantly modified. Nested flux surfaces inside the island lead to a good electron confinement which creates a potential well inside the island. Electrons near the core of the plasma (near the island X-point) are expected to undergo a rapid radial transport moderated by collisions, along the magnetic field lines near the island boundary, especially within one $\rho_{e}$ of the island separatrix. This enhanced preferential electron flux from the core leads to the development of an ambipolar radial electric field, which is positive near the core. This simple model is supported by our experimental evidence of much reduced radial electric field structure in the vicinity of the islands when the island width or the neutral collisions are increased. The tendency of the plasma profiles to follow flux surfaces, including within islands, is a somewhat surprising in view of the short mean-free-path relative to the circumference.

\section{ACKNOWLEDGMENTS}

The authors would like to thank Michael Shats (ANU, Canberra, Australia) and Chris Hegna (UW-Madison) for valuable discussions and $\mathrm{H}-1 \mathrm{NF}$ team for experimental operations. This work was performed on the H-1NF National Plasma Fusion Research Facility established by the Australian Government and operated by the Australian National University. This research was supported in part by the Australian Research Council Grant No. DP0344361 and the U.S. Department of Energy under Contract No. DE-AC0500OR22725 with UT-Battelle, LLC.

${ }^{1}$ A. Iiyoshi, A. Komori, A. Ejiri, M. Emoto, H. Funaba, M. Goto, K. Ida, H. Idei, S. Inagaki, S. Kado, O. Kaneko, K. Kawahata, T. Kobuchi, S. Kubo, R. Kumazawa, S. Masuzaki, T. Minami, J. Miyazawa, T. Morisaki, S. Morita, S. Murakami, S. Muto, T. Mutoh, Y. Nagayama, Y. Nakamura, H. Nakanishi, K. Narihara, K. Nishimura, N. Noda, S. Ohdachi, N. Ohyabu, Y. Oka, M. Osakabe, T. Ozaki, B. Peterson, A. Sagara, S. Sakakibara, R. Sakamoto, H. Sasao, M. Sasao, K. Sato, M. Sato, T. Seki, T. Shimozuma, M. Shoji, H. Suzuki, Y. Takeiri, K. Tanaka, K. Toi, T. Tokuzawa, K. Tsumori, K. Tsuzuki, K. Watanabe, T. Watari, H. Yamada, I. Yamada, S. Yamaguchi, M. Yokoyama, R. Akiyama, H. Chikaraishi, K. Haba, S. Hamaguchi, M. Iima, S. Imagawa, N. Inoue, K. Iwamoto, S. Kitagawa, J. Kodaira, Y. Kubota, R. Maekawa, T. Mito, T. Nagasaka, A. Nishimura, C. Takahashi, K. Takahata, Y. Takita, H. Tamura, T. Tsuzuki, S. Yamada, K. Yamauchi, N. Yanagi, H. Yonezu, Y. Hamada, K. Matsuoka, K. Murai, K. Ohkubo, I. Ohtake, M. Okamoto, S. Satoh, T. Satow, S. Sudo, S. Tanahashi, K. Yamazaki, M. Fujiwara, and O. Motojima, Nucl. Fusion 39, 1245 (1999).

${ }^{2}$ N. Ohyabu, K. Narihara, H. Funaba, T. Morisaki, S. Masuzaki, K. Kawahata, A. Komori, O. Kaneko, H. Yamada, P. deVries, M. Emoto, M. Goto, Y. Hamada, K. Ida, H. Idei, S. Inagaki, N. Inoue, S. Kado, S. Kubo, R. Kumazawa, T. Minami, J. Miyazawa, S. Morita, S. Murakami, T. Mutoh, S. Muto, and Y. Nagayama, Phys. Rev. Lett. 84, 103 (2000).

${ }^{3}$ M. Yokoyama, H. Maaßberg, C. D. Beidler, V. Tribaldos, K. Ida, F. Castejn, T. Estrada, A. Fujisawa, T. Minami, T. Shimozuma, Y. Takeiri, J. Herranz, S. Murakami, and H. Yamada, Fusion Sci. Technol. 50, 327 (2006).

${ }^{4}$ M. Yokoyama, H. Maaßberg, C. Beidler, V. Tribaldos, K. Ida, T. Estrada, F. Castejon, A. Fujisawa, T. Minami, T. Shimozuma, Y. Takeiri, A. Dinklage, S. Murakami, and H. Yamada, Nucl. Fusion 47, 1213 (2007).

${ }^{5}$ Y. Nagayama, K. Narihara, Y. Narushima, N. Ohyabu, T. Hayashi, K. Ida, S. Inagaki, D. Kalinina, R. Kanno, A. Komori, T. Morisaki, R. Sakamoto,
S. Sudo, N. Tamura, T. Tokuzawa, H. Yamada, M. Yoshinuma, and L. Experimental Group, Nucl. Fusion 45, 888 (2005).

${ }^{6}$ T. Oikawa, A. Isayama, T. Fujita, T. Suzuki, T. Tuda, and G. Kurita, Phys. Rev. Lett. 94, 125003 (2005).

${ }^{7}$ N. Ohyabu, K. Ida, T. Morisaki, K. Narihara, A. Komori, K. Watanabe, Y. Narushima, Y. Nagayama, M. Shoji, N. Ashikawa, M. Emoto, H. Funaba, M. Goto, H. Idei, K. Ikeda, S. Inagaki, N. Inoue, M. Isobe, K. Khlopenkov, T. Kobuchi, A. Kostrioukov, S. Kubo, R. Kumazawa, Y. Liang, S. Masuzaki, T. Minami, and J. Miyazawa, Phys. Rev. Lett. 88, 055005 (2002).

${ }^{8}$ S. Inagaki, N. Tamura, K. Ida, Y. Nagayama, K. Kawahata, S. Sudo, T. Morisaki, K. Tanaka, and T. Tokuzawa, Phys. Rev. Lett. 92, 055002 (2004).

${ }^{9}$ A. Weller, A. D. Cheetham, A. W. Edwards, R. D. Gill, A. Gondhalekar, R. S. Granetz, J. Snipes, and J. A. Wesson, Phys. Rev. Lett. 59, 2303 (1987).

${ }^{10}$ P. C. D. Vries, A. J. H. Donne, S. H. Heijnen, C. A. J. Hugenholtz, A. Kramer-Flecken, F. C. Schuller, and G. Waidmann, Nucl. Fusion 37, 1641 (1997).

${ }^{11}$ S. M. Hamberger, B. D. Blackwell, L. E. Sharp, and D. B. Shenton, Fusion Technol. 17, 123 (1990).

${ }^{12}$ J. H. Harris, L. Cantrell, T. C. Hender, B. A. Carreras, and R. N. Morris, Nucl. Fusion 25, 623 (1985).

${ }^{13}$ S. T. A. Kumar, B. D. Blackwell, and J. H. Harris, Rev. Sci. Instrum. 78, 013501 (2007).

${ }^{14}$ S. T. A. Kumar, B. D. Blackwell, and J. H. Harris, Nucl. Fusion 49, 035001 (2009)

${ }^{15}$ M. G. Shats and W. M. Solomon, New J. Phys. 4, 30 (2002).

${ }^{16}$ N. Hershkowitz, Plasma Diagnostics, edited by O. Auciello and D. L. Flamm (Academic, San Diego, CA, 1989), Vol. 1.

${ }^{17}$ J. Howard, C. Michael, F. Glass, and A. Danielsson, Plasma Phys. Controlled Fusion 45, 1143 (2003)

${ }^{18}$ C. A. Michael, "Doppler spectroscopy of Argon plasmas in H-1NF using a coherence imaging camera," Ph.D. thesis, The Australian National University, 2003.

${ }^{19}$ C. A. Michael, J. Howard, and B. D. Blackwell, Phys. Plasmas 11, 4008 (2004).

${ }^{20}$ M. G. Shats, D. L. Rudakov, R. W. Boswell, and G. G. Borg, Phys. Plasmas 4, 3629 (1997).

${ }^{21}$ J. Harris, M. Shats, B. Blackwell, W. Solomon, D. Pretty, S. Collis, J. Howard, H. Xia, C. Michael, and H. Punzmann, Nucl. Fusion 44, 279 (2004).

${ }^{22}$ W. L. Pickles, M. O. Calderon, M. R. Carter, C. A. Clower, R. P. Drake, A. L. Hunt, D. Lang, T. C. Simonen, and W. C. Turner, J. Vac. Sci. Technol. A 1, 1288 (1983).

${ }^{23}$ H. Punzmann, M. G. Shats, W. M. Solomon, and H. Xia, Rev. Sci. Instrum. 74, 2048 (2003).

${ }^{24}$ Á. Yanguas-Gil, J. Cotrino, and L. L. Alves, J. Phys. D: Appl. Phys. 38, 1588 (2005)

${ }^{25}$ M. G. Shats, W. M. Solomon, and J. N. Talmadge, J. Plasma Fusion Res. Ser. 4, 219 (2001).

${ }^{26}$ K. Ida, S. Inagaki, N. Tamura, T. Morisaki, N. Ohyabu, K. Khlopenkov, S. Sudo, K. Watanabe, M. Yokoyama, T. Shimozuma, Y. Takeiri, K. Itoh, M. Yoshinuma, Y. Liang, K. Narihara, K. Tanaka, Y. Nagayama, T. Tokuzawa, K. Kawahata, H. Suzuki, A. Komori, T. Akiyama, N. Ashikawa, M. Emoto, H. Funaba, P. Goncharov, M. Goto, H. Idei, K. Ikeda, M. Isobe, O. Kaneko, H. Kawazome, T. Kobuchi, A. Kostrioukov, S. Kubo, R. Kumazawa, S. Masuzaki, T. Minami, J. Miyazawa, S. Morita, S. Murakami, S. Muto, T. Mutoh, Y. Nakamura, H. Nakanishi, Y. Narushima, K. Nishimura, N. Noda, T. Notake, H. Nozato, S. Ohdachi, Y. Oka, M. Osakabe, T. Ozaki, B. Peterson, A. Sagara, T. Saida, K. Saito, S. Sakakibara, R. Sakamoto, M. Sasao, K. Sato, M. Sato, T. Seki, M. Shoji, N. Takeuchi, K. Toi, Y. Torii, K. Tsumori, T. Watari, Y. Xu, H. Yamada, I. Yamada, S. Yamamoto, T. Yamamoto, Y. Yoshimura, I. Ohtake, K. Ohkubo, T. Mito, T. Satow, T. Uda, K. Yamazaki, K. Matsuoka, O. Motojima, and M. Fujiwara, Nucl. Fusion 44, 290 (2004).

${ }^{27}$ K. Ida, M. Yoshinuma, M. Yokoyama, S. Inagaki, N. Tamura, B. Peterson, T. Morisaki, S. Masuzaki, A. Komori, Y. Nagayama, K. Tanaka, K. Narihara, K. Watanabe, C. Beidler, and L. Experimental Group, Nucl. Fusion 45, 391 (2005).

${ }^{28}$ L. G. Askinazi, V. E. Golant, V. A. Kornev, S. V. Lebedev, A. S. Tukachinsky, M. I. Vildjunas, and N. A. Zhubr, Plasma Phys. Controlled Fusion 48, A85 (2006). 
${ }^{29}$ K. C. Shaing, C. C. Hegna, J. D. Callen, and W. A. Houlberg, Nucl. Fusion 43, 258 (2003).

${ }^{30}$ F. F. Chen, Introduction to Plasma Physics (Plenum, New York, 1977).

${ }^{31} \mathrm{~F}$. Glass, "Tomographic visible spectroscopy of plasma emissivity and ion temperatures," Ph.D. thesis, The Australian National University, 2004.

${ }^{32}$ F. Glass, J. Howard, and B. Blackwell, IEEE Trans. Plasma Sci. 33, 472 (2005).
${ }^{33}$ D. D. Blackwell and F. F. Chen, Plasma Sources Sci. Technol. 6, 569 (1997).

${ }^{34}$ T. Shimozuma, S. Kubo, H. Idei, S. Inagaki, N. Tamura, T. Tokuzawa, T. Morisaki, K. Watanabe, K. Ida, I. Yamada, K. Narihara, S. Muto, M. Yokoyama, Y. Yoshimura, T. Notake, K. Ohkubo, T. Seki, K. Saito, R. Kumazawa, T. Mutoh, T. Watari, A. Komori, and The LHD Experimental Group, Nucl. Fusion 45, 1396 (2005). 